\title{
Malignant neurilemmoma of the lung metastasizing to the heart
}

\author{
N. F. CROFTS AND G. B. FORBES \\ From Keycol Hospital, Sittingbourne, and the Department of Pathology, Kent and Canterbury \\ Hospital
}

Primary neurogenic tumours of the lung are rare in the absence of generalized neurofibromatosis. Neilson (1958) collected 25 reports from the world literature up to 1957 , but histological confirmation was lacking or incomplete in 18 . To the seven cases which Neilson regarded as fulfilling the criteria necessary for precise histological diagnosis he added one of his own; this was a neurogenic sarcoma removed surgically with the left lower lobe from a 35-year-old man. The diagnoses in the other confirmed cases were neurogenic sarcoma (2), benign neurilemmoma (3), and benign neurofibroma (2). . None of the neurogenic sarcomata produced metastases.

The case presented in this paper is of especial interest in that the primary lung tumour, histologically a neurilemmoma, was complicated by a large secondary deposit in the heart.

\section{CASE REPORT}

A woman of 59 , employed as a typist, attended a chest clinic on 16 February 1962 complaining of intermittent bronchitis for 10 years, and becoming more frequent in the preceding six months. There had been no haemoptyses. She was dyspnoeic when walking on the level and had lost half a stone $(3 \cdot 2$ kg.) in weight recently. She had smoked 10 cigarettes a day for many years.

Her nutritional state was good. There was no evidence of generalized neurofibromatosis. She was afebrile. There was no clubbing of the fingers. The breath sounds were diminished and the percussion note was dull at the right lung base laterally and posteriorly. There were no adventitious sounds.

The heart rhythm was regular. There was a soft apical systolic murmur. The jugular venous pressure was not raised. The blood pressure was $170 / 90 \mathrm{~mm}$. $\mathrm{Hg}$. There were no other abnormal signs.

Chest radiographs showed a large rounded opacity in the right lower lobe (Figs. 1a and b). Fluoroscopy revealed slightly restricted movements of the right diaphragm. On 27 February bronchoscopy was performed. The right lower lobe orifice below the cardiac segment was almost completely stenosed by pressure from outside. A bronchial biopsy specimen was taken but histological examination revealed no evidence of neoplasia. In spite of this, bronchial carcinoma was strongly suspected on clinical and bronchoscopic grounds.

One week after bronchoscopy the patient became more dyspnoeic and developed tachycardia. On 19 March a radiograph showed medium-sized bilateral pleural effusions. These were thought to be due to secondary carcinoma of the pleura. Plans for surgical treatment were abandoned and she was discharged home. Two weeks later she was readmitted to hospital because of severe dyspnoea. Her condition was poor. Orange fluid (36 fluid oz. (1,026 ml.)) was aspirated from the left pleural cavity and $45 \mathrm{mg}$. of thiotepa was injected intrapleurally. Her breathing improved after this and she was comfortable for the next month. She then became more breathless, but obtained relief when sitting up and leaning forwards. On 12 June 19628 fluid oz. (228 ml.) of bloodstained fluid was aspirated from the right pleural cavity and a needle biopsy of the mass in the right lower lobe was performed. The section appeared to consist of fibrous tissue only. After this she gradually became weaker. She remained afebrile but her pulse was rapid (120/minute).

She died on 25 June 1962 about four months after her first attendance at the clinic.

NECROPSY The lower lobe of the right lung was $D$ almost completely replaced by a rounded greyish- 을 white fibrous neoplasm measuring $9 \mathrm{~cm}$. in diameter $\bar{N}$ (Fig. 2). The cut surface was of firm texture and 5 slightly whorled, and there were haemorrhagic areas $N$ in the central part of the tumour. The external appearance of the heart was normal, but on opening the organ a large ovoid tumour was found in the left atrium; it measured $7 \mathrm{~cm}$. in main diameter and was of similar gross appearance to the lung tumour (Fig. 3). It filled the chamber and was attached to the postero-medial wall by a thin short pedicle.

The diaphragm was normal and no other significant abnormalities were found.

HISTOLOGY The pulmonary tumour was highly cellular and consisted of elongated fibroblast-like cells with spindle-shaped nuclei. The latter were grouped in 
FIG. 1a and b. Postero-anterior and right lateral radiographs showing the tumour in the right lower lobe.

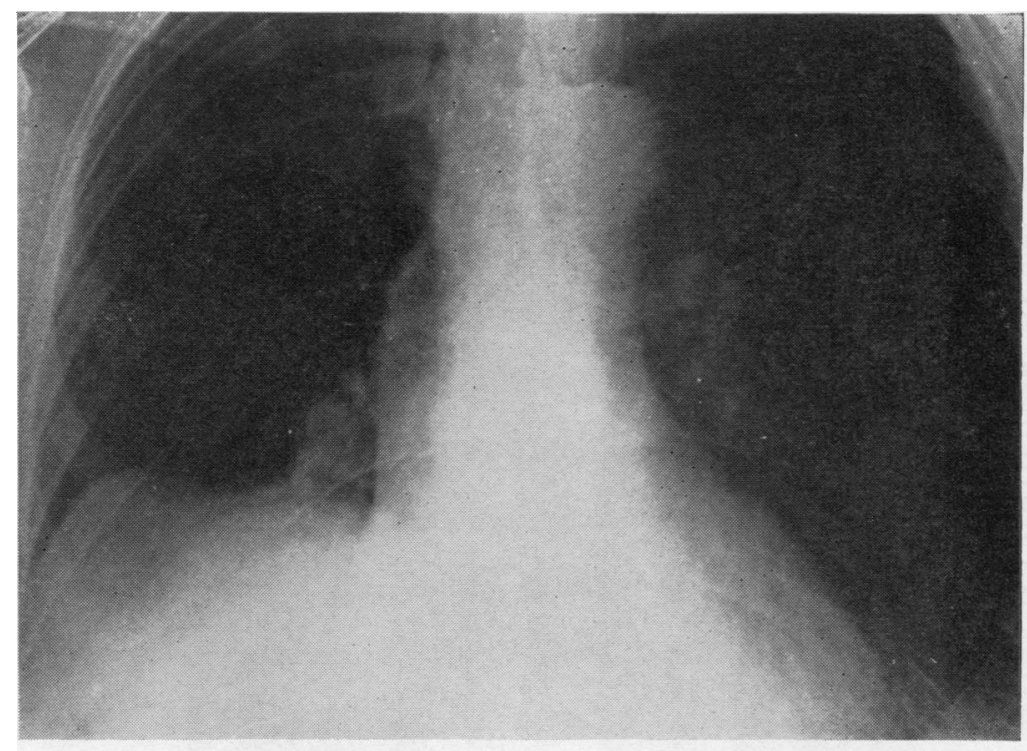

(a)

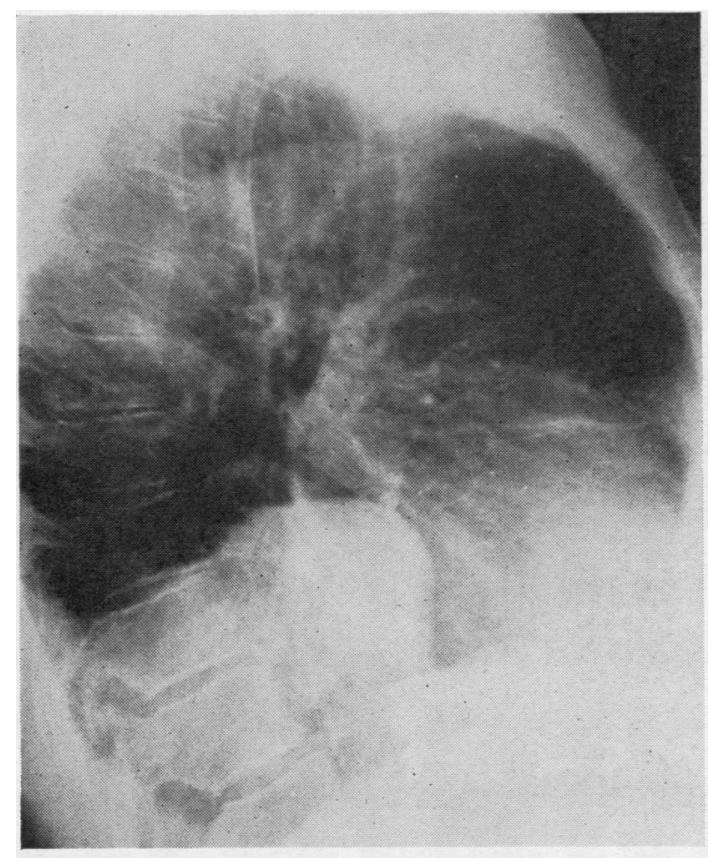

(b)

parallel rows, producing a striking pattern of nuclear regimentation with palisade formation (Figs. 4a and b). The cells were for the most part uniform and well differentiated, but a few showed nuclear aberration and mitotic activity. The tumour had a fairly well-defined border and was contained within a pseudo-capsule of fibrous tissue ; in part it was rather vascular. There was a heavy network of coarse, darkstaining reticulin fibrils running in long parallel strands between, and to a lesser extent, around the cells. There were also scanty strands of collagen. Myofibrils were not demonstrable in phosphotungstic acid-haematoxylin-stained sections, and the tinctorial reactions with trichrome and van Gieson stains were not those of a smooth muscle tumour.

The nuclear regimentation, which was such a prominent feature in the lung tumour, was not reproduced to the same extent in the cardiac tumour. In the latter situation, the fibroblast-like cells were arranged in fascicles with little tendency to form palisades (Fig. 5). In other respects, however, there was a strong histological resemblance between the two tumours, and they reacted tinctorially with a variety of stains in an identical fashion.

\section{DISCUSSION}

It is unfortunate that these tumours were not diagnosed during life, as there would have been a possibility of resection. An intrapulmonary tumour complicated by blood-stained pleural effusions in a smoker, aged 60 , strongly suggests an inoperable carcinoma of the bronchus. Thoracotomy in this case was further prevented by the patient's poor general condition.

The intra-atrial tumour was not suspected during life. In retrospect it is probable that it caused the orthopnoea by raising the left atrial pressure. 


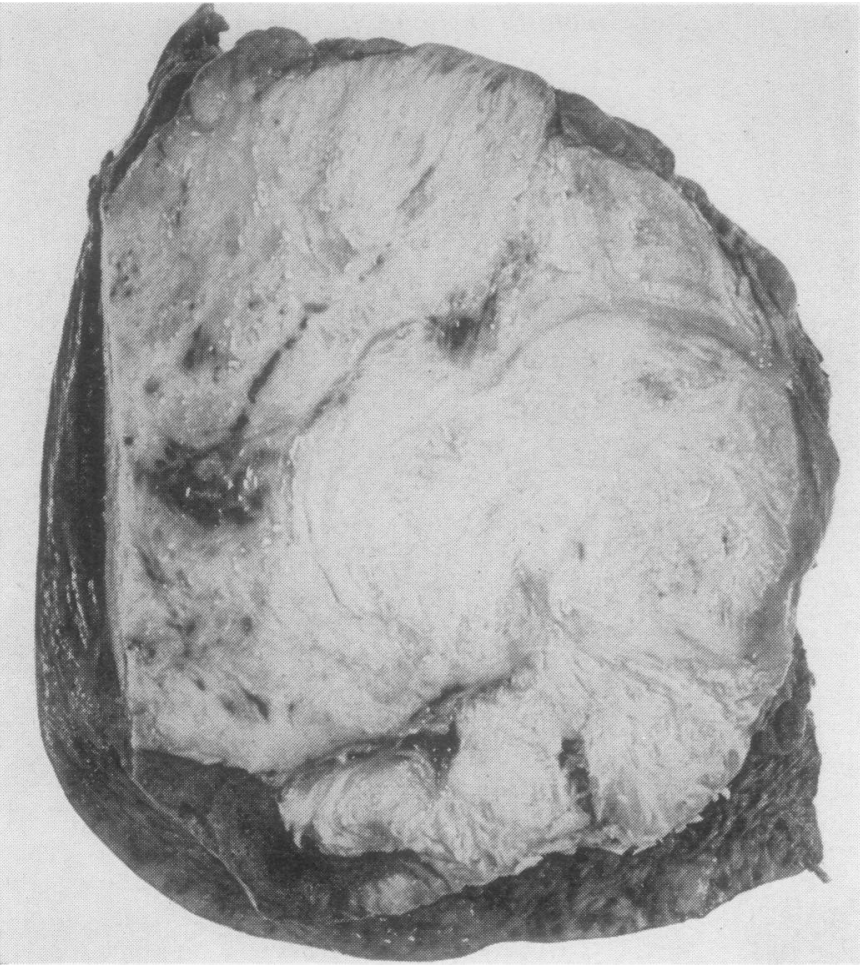

FIG. 2

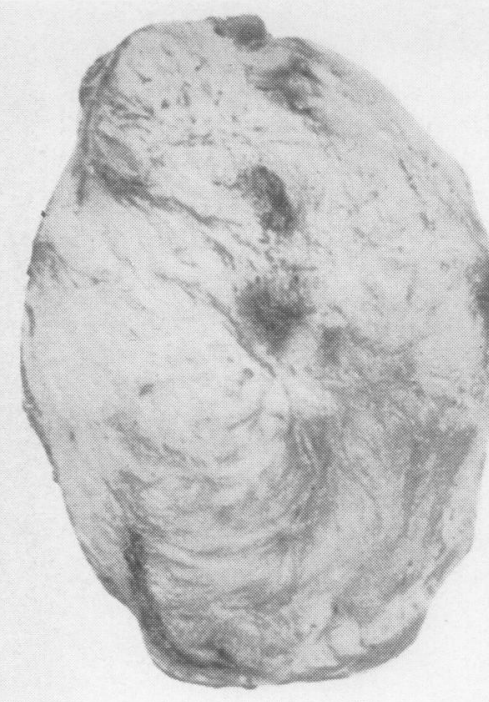

FIG. 3

FIG. 2. The pulmonary tumour (natural size). FIG. 3. The cardiac tumour (natural size). 응

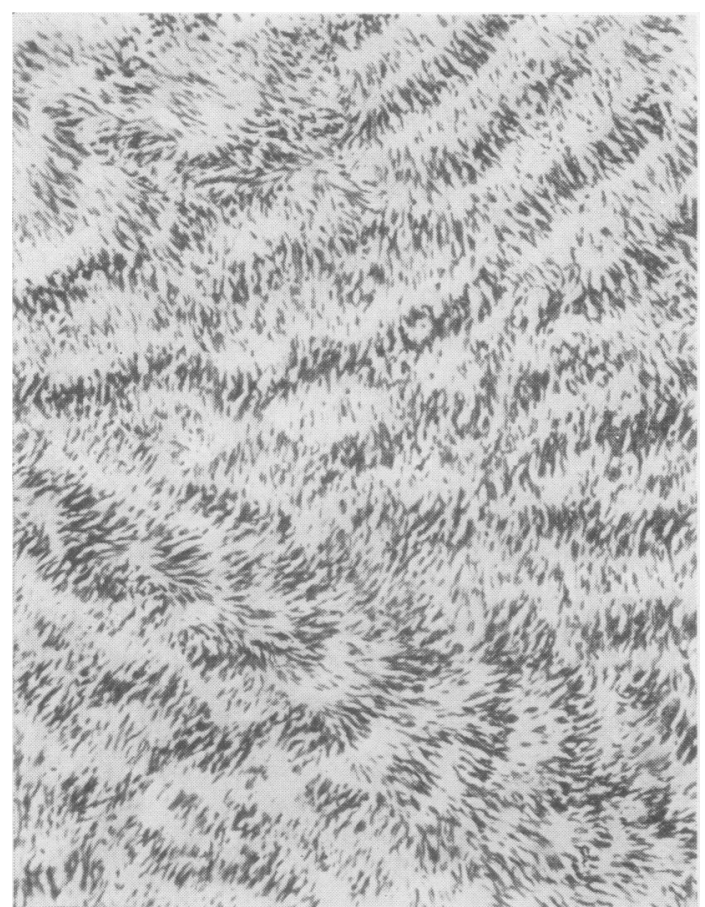

FIG. 4a. The lung tumour showing nuclear palisading. $\times 80$.

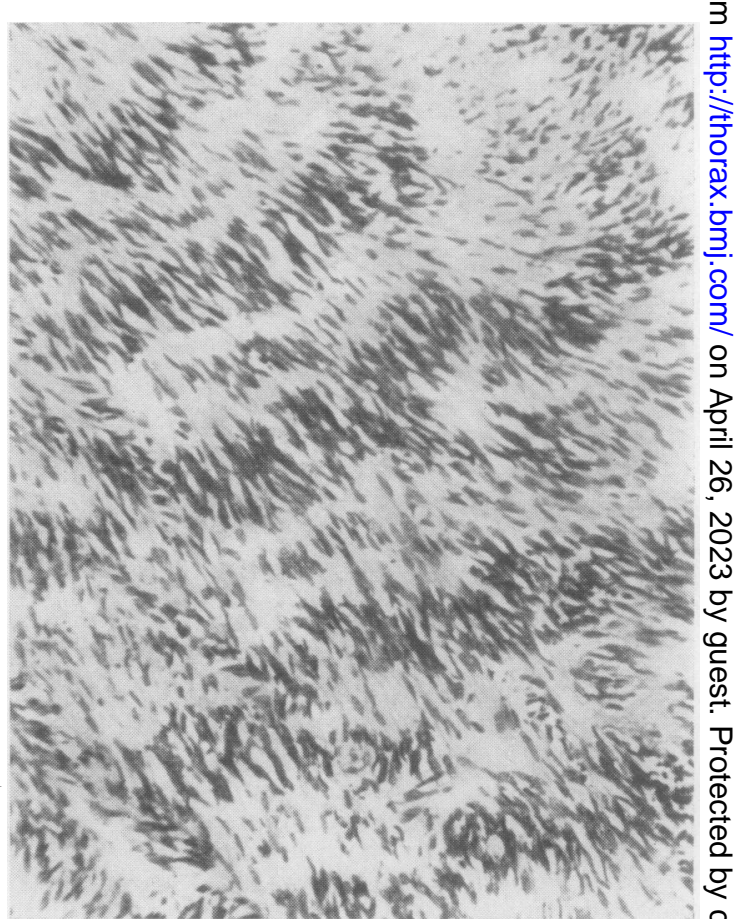

FIG. 4b. Lung tumour. Detail of nuclear palisading.음 $\times 140$. 


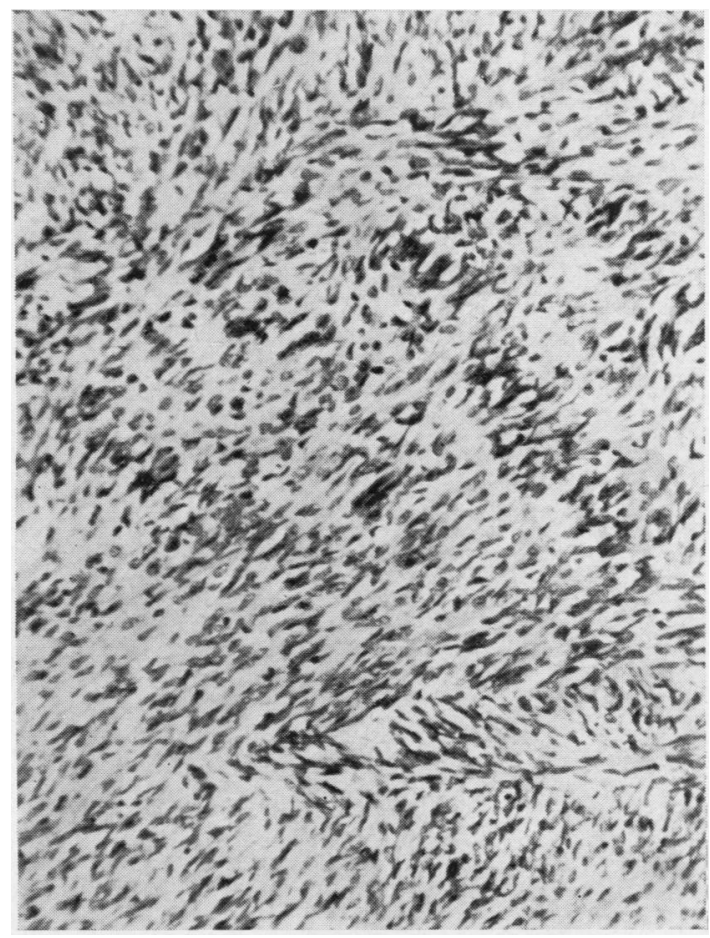

FIG. 5. The cardiac tumour. $\times 140$.

When examined microscopically, the pulmonary tumour showed the typical cytological pattern of a neurilemmoma (schwannoma) composed of Antoni type A tissue. The characteristic nuclear palisading was the predominant feature in sections taken from widely separated parts of the tumour, and the photomicrographs shown in Figs. 4a and $b$ are representative of the tumour as a whole. Leiomyomata may also show nuclear palisading but not to this degree; moreover, the staining reactions were not those of a smooth muscle tumour. The cardiac tumour was less well differentiated, but there was sufficient similarity on naked-eye and microscopic examination to indicate that the two tumours had a common histogenesis. The relative size of the tumours and the normal direction of blood flow from the lung to the left atrium leave little room for doubt that the tumour in the lung was the primary growth and the heart lesion a metastatic deposit. The pulmonary tumour probably arose from sympathetic nerve fibres accompanying arterioles or bronchioles; these fibres are non-medullated but possess a neurilemma (Schwann cells). Presum. ably the intra-atrial tumour was the result of endocardiac implantation of tumour emboli which had travelled to the heart via the pulmonary veins.

It could be argued that the tumours were separate primaries growing simultaneously and independently. However, in this event the cardiac tumour would have arisen from the cardiac plexus and would then have been situated not in the atrial chamber but on the external aspect of the base of the heart or in the myocardium, as in the case described by Dammert, Elfving, aud Halonen (1955).

Tumours composed wholly or largely of neoplastic Schwann cells are almost invariably benign. The criteria for regarding the present case as malignant are the presence of the intra-cardiac secondary growth, the demonstration of nuclear aberration and mitotic activity in a highly cellular growth, and the rapid deterioration in the patient's general physical condition accompanied by loss of weight.

It is well known that tumours of peripherail neurogenic derivation, other than neurilemmoma, sometimes develop malignant characters and may even metastasize to distant organs. These are referred to collectively as neurogenic sarcoma or as malignant neurofibroma when complicating von Recklinghausen's disease. Areas of neurilemmomatous differentiation are seen occasionally in this type of neoplasm (Willis, 1960), and it may be that the tumour which we have studied was a neurogenic sarcoma showing an extreme degree of neurilemmomatous differentiation. None the less, we consider that the term 'malignant neurilemmoma' is both warranted and preferable in the present instance because of the distinctive histological pattern throughout the primary tumour.

\section{SUMMARY}

A case of malignant neurilemmoma of the lung with a secondary tumour in the left atrium of the heart is described. It is believed to be unique in the medical literature.

We wish to thank Dr. N. J. H. Davidson, who performed the necropsy, Dr. A. Golebiowski, who performed the bronchoscopy, Dr. K. F. W. Hinson for his help and interest, and Mr. E. W. Spice for the photographs.

\section{REFERENCES}

Dammert, K., Elfving, G., and Halonen, P. I. (1955). Neurogenic sarcoma in the heart. Amer. Heart J., 49, 794

Neilson, D. B. (1958). Primary intrapulmonary neurogenic sarcoma. J. Path. Bact., 76, 419.

Willis, R. A. (1960). Pathology of Tumours, 3rd ed., Ch. 54. Butterworths, London. 